\title{
ON THE GEOMETRY OF OUTER SPACE
}

\author{
KAREN VOGTMANN
}

Abstract. Outer space is a space of graphs used to study the group $\operatorname{Out}\left(F_{n}\right)$ of outer automorphisms of a finitely generated free group. We discuss an emerging metric theory for Outer space and some applications to $\operatorname{Out}\left(F_{n}\right)$.

\section{INTRODUCTION}

Outer space was introduced in the early 1980s as a tool for studying the group $\operatorname{Out}\left(F_{n}\right)$ of outer automorphisms of a finitely generated free group [16]. It is a contractible space on which $\operatorname{Out}\left(F_{n}\right)$ acts with finite stabilizers, and it should be thought of as analogous to a symmetric space with the action of a non-uniform lattice or to the Teichmüller space of a surface with the action of the mapping class group of the surface. Outer space also has close connections to many other areas of mathematics, from Kontsevich's graph homology theory to modular forms, tropical geometry, and the mathematics of phylogenetic trees.

Outer space is a parameter space for certain objects equipped with metrics, but historically the space itself and its quotient by $\operatorname{Out}\left(F_{n}\right)$ have been studied mostly by topological and combinatorial methods. These methods have yielded (and continue to yield) many new results about $\operatorname{Out}\left(F_{n}\right)$, including information about its finiteness properties, subgroup structure, algorithmic properties, and cohomology. Striking parallels have emerged between $\operatorname{Out}\left(F_{n}\right)$ and both lattices and mapping class groups, and we would like to understand how far these parallels extend. An example particularly relevant to geometric group theory is the question of whether a group which has (approximately) the same geometry as Out $\left(F_{n}\right)$ must be (almost) isomorphic to $\operatorname{Out}\left(F_{n}\right)$; the technical term is whether $\operatorname{Out}\left(F_{n}\right)$ is quasi-isometrically rigid. In the cases of lattices and mapping class groups, quasi-isometric rigidity and other results were established using the geometry of symmetric spaces and Teichmüller spaces in essential ways.

Outer space is not a manifold, so some care is required when attempting to use geometric tools to study it. In the past few years a metric theory of Outer space has begun to emerge based on a natural non-symmetric metric. The resulting geometric point of view is yielding new information about $\operatorname{Out}\left(F_{n}\right)$ as well as elegant new proofs and better understanding of older results, and it is strengthening the analogy between Outer space and the classical theories of symmetric spaces and Teichmüller spaces.

Many people are now contributing to the geometric theory of Outer space, and I have provided a cursory guide to some of the literature in the last section. In this short article I have chosen to sketch primarily the work of Yael Algom-Kfir,

Received by the editors May 31, 2014.

2010 Mathematics Subject Classification. Primary 20F65. 
Mladen Bestvina, and Mark Feighn. For the interested reader, a more thorough introduction to their work can be found in Bestvina's lecture notes from his Summer 2012 course at the Park City Mathematics Institute [7.

\section{Definitions of Outer space}

For the most part we will think of Outer space as a space of marked metric graphs, where the metric on a graph is specified by giving each edge a positive real length, and the marking identifies the fundamental group of the graph with the free group $F_{n}$. However, there are several equivalent ways to define Outer space, and we will begin with the one which is quickest to state. This is in terms of actions of $F_{n}$ on metric simplicial trees, i.e., contractible one-dimensional simplicial complexes metrized so that each edge is isometric to an interval of the real line. An action is called minimal if it has no invariant subtree. Using this concept, we can give a succinct definition of Outer space, though we will temporarily duck the question of what topology to put on it.

Definition 2.1. (Unprojectivized) Outer space in rank $n$ is the space of free minimal actions of $F_{n}$ by isometries on metric simplicial trees.

Here two actions are considered the same if there is an equivariant isometry between the trees. The notation $\mathrm{CV}_{n}$ has become standard for this space. The projectivized version is denoted $\mathrm{CV}_{n}$, i.e., in $\mathrm{CV}_{n}$ two actions are equivalent if they differ only by scaling the tree's metric. Instead of taking equivalence classes, one can also think of a point in $\mathrm{CV}_{n}$ as normalized so that the "volume" of the quotient graph (i.e., the sum of its edge lengths) is one. In most of the remainder of this article this is the convention we will adopt.

The action of $\operatorname{Out}\left(F_{n}\right)$ on $\mathrm{CV}_{n}$ or $\mathrm{CV}_{n}$ is also easily described using this definition. Given a point $\rho: F_{n} \rightarrow \operatorname{Isom}(T)$ and an automorphism $\phi$, we get a new point by composing $\rho \circ \phi: F_{n} \rightarrow \operatorname{Isom}(T)$. This induces an action of $\operatorname{Out}\left(F_{n}\right)$ since inner automorphisms give equivariantly isometric actions, i.e., they fix all of $\mathrm{CV}_{n}$. Note that this is a right action, and acting by a group element does not affect the metric on the tree.

2.1. Definition in terms of graphs. Here is how to translate the definition of $\mathrm{CV}_{n}$ given above in terms of graphs. The graph corresponding to an action of $F_{n}$ on a tree $T$ is the quotient of $T$ by the action. The fact that we are only considering minimal actions implies that these quotient graphs are compact and have no univalent or bivalent vertices. If we choose a basis for $F_{n}$ and a basepoint for $T$, then arcs joining the basepoint to its images under the generators of $F_{n}$ descend to immersed loops in the quotient graph $\Gamma$. Designating these loops as the images of the petals of a rose $R_{n}$ gives a map $g: R_{n} \rightarrow \Gamma$ called a marking which identifies $\pi_{1}\left(R_{n}\right) \equiv F_{n}$ with $\pi_{1}(\Gamma)$ (see Figure 1). The fact that equivariantly isometric trees are the same point in $\mathrm{CV}_{n}$ can be stated in terms of marked graphs by defining $(g, \Gamma)$ and $\left(g^{\prime}, \Gamma^{\prime}\right)$ to be equivalent if there is an isometry $h: \Gamma \rightarrow \Gamma^{\prime}$ with $h \circ g$ homotopic to $g^{\prime}$. Since we do not want this definition to depend on the choice of basepoint, we do not require that this homotopy preserve basepoints.

An element $\phi \in \operatorname{Out}\left(F_{n}\right)$ acts by changing the marking, i.e., if we represent $\phi$ by a homotopy equivalence $f: R_{n} \rightarrow R_{n}$, then $(g, \Gamma) \cdot \phi=(g \circ f, \Gamma)$. 


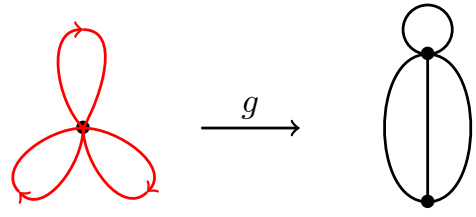

FiguRE 1. A marked graph

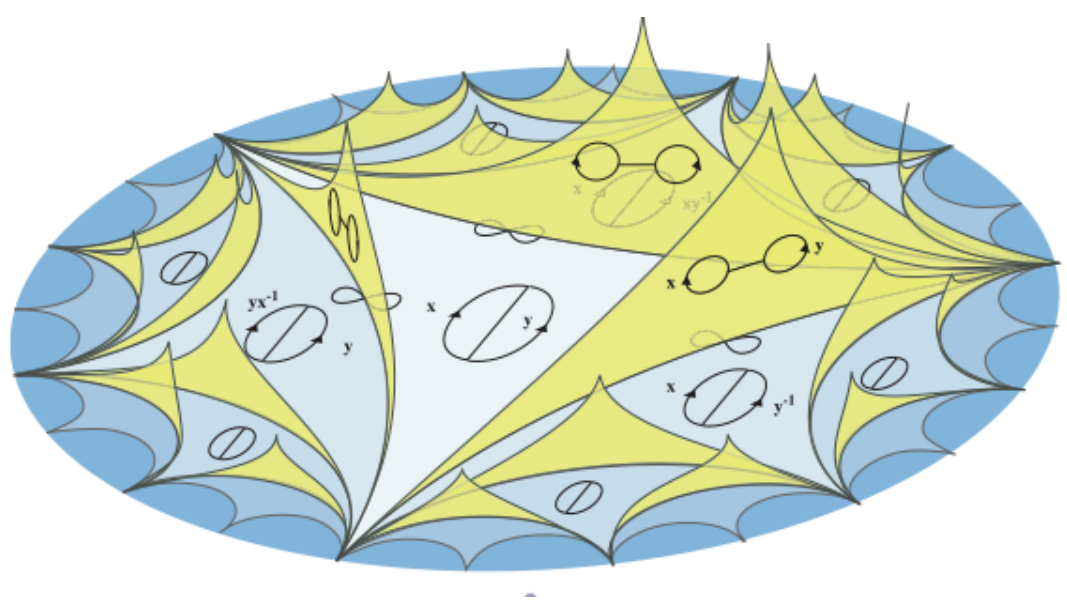

FiguRE 2. Outer space in rank 2

2.2. Simplicial decomposition and topology. The description of $\mathrm{CV}_{n}$ in terms of graphs makes it easy to decompose $\mathrm{CV}_{n}$ into a union of open simplices. Figure 2 is a picture of this decomposition for $n=2$. The simplex containing $(g, \Gamma)$ is formed by assigning all possible positive edge lengths to the edges of $\Gamma$, subject to the condition that the volume must be one. The simplex containing $\left(g^{\prime}, \Gamma^{\prime}\right)$ is a face of the one containing $(g, \Gamma)$ if $\left(g^{\prime}, \Gamma^{\prime}\right)$ can be obtained from $(g, \Gamma)$ by collapsing some edges of $\Gamma$ to points; the face of the simplex is obtained by assigning those edges length zero. Some faces of each simplex are missing, since you cannot collapse a loop without changing the rank of the fundamental group. If we formally add all of the "missing faces", we obtain a simplicial complex called the simplicial completion of Outer space. The simplices which are not in $\mathrm{CV}_{n}$ are said to be at infinity. In rank 2 all of the vertices are at infinity, as well as two edges of each "barbell" simplex. (We remark that the term "simplicial completion" is used slightly differently in [1, though the paper proves that her notion agrees with the one used here.)

There are several natural ways of topologizing $\mathrm{CV}_{n}$, but they are all equivalent to giving it the quotient topology which arises from its description as the disjoint union of open simplices modulo the above face relations.

2.3. Definition in terms of sphere systems. Outer space $\mathrm{CV}_{n}$ can also be described using spheres embedded in a doubled handlebody $M_{n}$ (i.e., $M_{n}$ is the connected sum of $n$ copies of $\left.S^{1} \times S^{2}\right)$. Since $\pi_{1}\left(M_{n}\right)=F_{n}$, any diffeomorphism of $M$ induces an (outer) automorphism of $F_{n}$. Laudenbach 31] showed that the 
kernel of the resulting map from the group $\pi_{0}\left(\operatorname{Diff}\left(M_{n}\right)\right)$ of isotopy classes of diffeomorphisms to $\operatorname{Out}\left(F_{n}\right)$ is a finite 2-group which acts trivially on isotopy classes of embedded spheres. Thus one may build a simplicial complex with an $\operatorname{Out}\left(F_{n}\right)$ action whose vertices are isotopy classes of embedded spheres. A set of $k$ such vertices spans a $k$-simplex if spheres representing the vertices can be embedded disjointly into $M_{n}$; this is called the sphere complex $\mathcal{S}_{n}$.

Remark 2.2. In the above definition, if we replace "isotopy classes of spheres embedded in a doubled handlebody $M_{n}$ " by "isotopy classes of simple closed curves embedded in a surface $S$ ", we obtain the usual definition of the curve complex $\mathcal{C}(S)$ associated to a surface of genus $g>1$.

Hatcher shows that $\mathrm{CV}_{n}$ embeds into the the sphere complex $\mathcal{S}_{n}$ as a union of open simplices, corresponding to sphere systems with simply connected complementary pieces [27. The marked graph corresponding to a sphere system is simply the graph dual to the spheres, with one vertex in each complementary piece and one edge intersecting each sphere. Barycentric coordinates on the simplex corresponding to the sphere system determine edge-lengths for the graph, and the marking comes from the embedding of the graph into $M_{n}$. The open simplices coincide with those we described in the last section.

The sphere system model has advantages over other models, including the fact that different points of $\mathrm{CV}_{n}$ are represented by sets of spheres in the same manifold, giving rise to natural ways of comparing them (e.g., counting the number of intersection circles), natural paths between them (called surgery paths), and the natural way of completing Outer space to a simplicial complex (the sphere complex).

\section{The Lipschitz Metric ON OUter SPACE}

Thurston studied a non-symmetric metric on Teichmüller space which measures the infimum of Lipschitz constants for a homotopy class of homeomorphisms from one hyperbolic surface to another [40]. He proved basic properties of this metric, showed that one can measure distance by looking at how much curves are stretched, described geodesics, and used the metric to give coordinates for Teichmüller space. Bestvina was perhaps the first to suggest transporting this idea to Outer space, and a nice account of the basic properties of this metric can be found in his 2012 PCMI lecture notes 7 , where he attributes some of these as unpublished results of his former student Tad White. Francaviglia and Martino published the first systematic study of this metric in [17].

3.1. Definition of the metric. Let $f: X \rightarrow X^{\prime}$ be a map of metric spaces. Recall that the Lipschitz constant (or, more informally, the maximal stretch) of $f$ is

$$
L(f)=\sup _{x, y \in X} \frac{d_{X^{\prime}}(f(x), f(y))}{d_{X}(x, y)} .
$$

If $X$ is compact, we may replace sup with max in this definition.

Definition 3.1. The Lipschitz distance between two points $(g, \Gamma)$ and $\left(g^{\prime}, \Gamma^{\prime}\right)$ of $\mathrm{CV}_{n}$ is

$$
d\left((g, \Gamma),\left(g^{\prime}, \Gamma^{\prime}\right)\right)=\inf _{f} \log (L(f)),
$$

where the infimum is taken over all $f: \Gamma \rightarrow \Gamma^{\prime}$ with $f \circ g \simeq g^{\prime}$. 


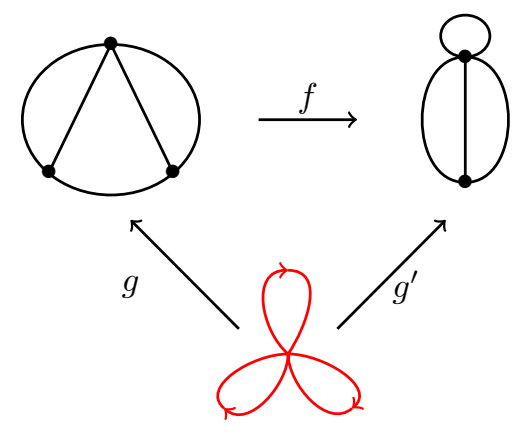

Figure 3. A difference of markings $f$

Since $\Gamma$ and $\Gamma^{\prime}$ are compact, the Arzelà-Ascoli theorem says that the limit of a minimizing sequence exists, so we may replace inf with min in this definition.

We can decrease the Lipschitz constant of a map $f: \Gamma \rightarrow \Gamma^{\prime}$ by tightening to make the restriction of $f$ to each edge of $\Gamma$ a linear immersion, i.e., any lift $\tilde{f}: \widetilde{\Gamma} \rightarrow \widetilde{\Gamma}^{\prime}$ of $f$ to universal covers maps edges of $\widetilde{\Gamma}$ linearly to arcs in $\widetilde{\Gamma}^{\prime}$. Thus for the purposes of computing the Lipschitz distance between two points of $\mathrm{CV}_{n}$, we need only consider maps with this property. Such a map $f$ is called a difference of markings from $(g, \Gamma)$ to $\left(g^{\prime}, \Gamma^{\prime}\right)$ if $f \circ g \simeq g^{\prime}$ (see Figure 3). Note that if two points of Outer space are in the same open simplex, then there is a difference of markings which is combinatorially the identity map.

In the rest of this article we will usually omit the marking when denoting a point of $\mathrm{CV}_{n}$, referring to $(g, \Gamma)$ simply as $\Gamma$ unless it is strictly necessary to specify the marking.

3.2. Calculating the Lipschitz distance. Although the definition of Lipschitz distance involves taking the infimum over an infinite family of maps, it turns out that in practice it is very easy to calculate the distance by a finite process using any map in the family. To explain this we first need a few definitions.

A map $f: \Gamma \rightarrow \Gamma^{\prime}$ with minimal Lipschitz constant in its homotopy class is called an optimal map. Since $\Gamma$ and $\Gamma^{\prime}$ are compact, optimal maps exist by the Arzelà-Ascoli theorem. For any optimal map $f: \Gamma \rightarrow \Gamma^{\prime}$ the tension subgraph $\Delta(f)$ is the subgraph of $\Gamma$ spanned by all edges which are stretched by exactly $L(f)$. A direction at a point $x$ in a graph $\Gamma$ is a germ of geodesic paths starting at $x$ (so there are two directions at most points, but the number of directions at a vertex is equal to the valence of the vertex).

Proposition $3.2\left(\right.$ [7, 17] ). Let $\Gamma$ and $\Gamma^{\prime}$ be two points in $\mathrm{CV}_{n}$, and let $f: \Gamma \rightarrow \Gamma^{\prime}$ be an optimal difference of markings with the additional condition that $\Delta(f)$ is minimal, i.e., there is no optimal $f^{\prime}$ homotopic to $f$ such that $\Delta\left(f^{\prime}\right)$ is a proper subgraph of $\Delta(f)$. Then

(1) $\Delta(f)$ is a core graph, i.e., it has no univalent vertices.

$(2) \Delta(f)$ has no vertex $v$ where all directions at $v$ map to the same direction at $f(v)$, and 
(3) $d\left(\Gamma, \Gamma^{\prime}\right)$ is equal to the supremum, over all loops $\alpha$ immersed in $\Gamma$, of

$$
\log \left(\frac{\operatorname{length}(f(\alpha))}{\operatorname{length}(\alpha)}\right)
$$

This proposition implies that we can measure the distance using any map homotopic to $f$ by measuring the shortest loop homotopic to $f(\alpha)$ for each immersed loop $\alpha$. This simplifies things considerably but still seems to involve looking at infinitely many loops. We observe, however, that the supremum in statement (3) must actually be realized on some loop which crosses each edge at most twice, since if a maximally stretched loop crosses an edge twice in the same direction it has a subloop with the same stretch. With a little more thought one can see that the supremum must be realized on either an embedded loop or an immersed loop which travels once around an embedded figure-eight or an embedded barbell (see Figure 4). Such loops are called candidate loops, and we see that we need only consider these (finitely many) candidate loops and their images to find the supremum. We collect these observations in the following corollary.

Corollary 3.3. The distance $d\left(\Gamma, \Gamma^{\prime}\right)$ can be computed from any difference of markings $f: \Gamma \rightarrow \Gamma^{\prime}$ by measuring the length of each candidate loop $\alpha$ in $\Gamma$ and the length of the shortest loop in the homotopy class of $f(\alpha)$.

3.3. Peculiarities of the Lipschitz distance. It is not difficult to show that the Lipschitz distance obeys two of the axioms for a metric. The triangle inequality follows because Lipschitz constants submultiply i.e., $L(g \circ f) \leq L(g) L(f)$. The fact that $d\left(\Gamma, \Gamma^{\prime}\right)=0$ if and only if $\Gamma=\Gamma^{\prime}$ follows because a surjective map between two volume 1 graphs which stretches nothing must be an isometry.

This distance function fails dramatically to be symmetric, however. A simple example is given in Figure 5, which shows two 2-petaled roses $\rho_{1}$ and $\rho_{2}$ in the same simplex of $\mathrm{CV}_{2}$. The first one, $\rho_{1}$, has edge lengths $\{1 / 2,1 / 2\}$, and $\rho_{2}$ has edge-lengths $\{\epsilon, 1-\epsilon\}$ for some small $\epsilon$. The combinatorial identity map $\rho_{1} \rightarrow \rho_{2}$ shrinks one edge and at most doubles the other, showing $d\left(\rho_{1}, \rho_{2}\right)<\log (2)$, while any map homotopic to the combinatorial identity in the opposite direction stretches the loop of length $\epsilon$ by at least $1 / 2 \epsilon$, so $d\left(\rho_{2}, \rho_{1}\right) \geq-\log (2 \epsilon)$. So this distance is not only asymmetric, there is no bound to the difference between $d(x, y)$ and $d(y, x)$. Note that the first rose is at the barycenter of its simplex, while the second is close to a missing face. This is symptomatic of the following general phenomenon:

You can get to the edge of Outer space very fast, but it will take you a long, long time to get back.
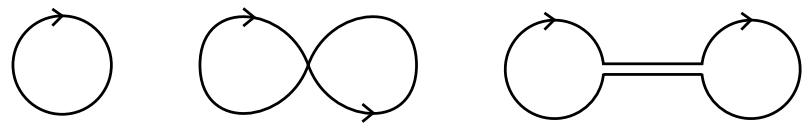

Figure 4. Candidate loops 


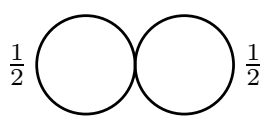

$\rho_{1}$

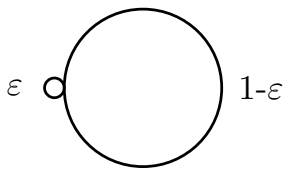

$\rho_{2}$

Figure 5. Asymmetry of the Lipschitz distance

\section{Using the Lipschitz MEtric to ClASSIFy ELEMENTS of $\operatorname{Out}\left(F_{n}\right)$}

The Lipschitz distance is invariant under the action of Out $\left(F_{n}\right)$ since the distance depends only on the difference of markings, i.e., Out $\left(F_{n}\right)$ acts on $\mathrm{CV}_{n}$ by isometries. In symmetric spaces, such as real hyperbolic space, isometries can be divided into three classes: those which are elliptic (which fix a point in the space), hyperbolic (translate a geodesic and act with North-South dynamics on the boundary of the space), and parabolic (neither of the above, but always fix a point on the boundary). Isometries of Teichmüller space exhibit the same three behaviors, and Bers used this fact to give a new proof of Thurston's classification of elements of the mapping class group 4. In particular, hyperbolic mapping classes are pseudo-Anosov, meaning they stretch the metric on the surface in one direction and shrink it in a complementary direction at all but finitely many points. This behavior had been codified by Thurston using a pair of complementary train tracks for a pseudo-Anosov homeomorphism, which gives a powerful tool for working with these mapping classes.

One property of pseudo-Anosov mapping classes is that they are irreducible, i.e., no proper subsurface is preserved. An outer automorphism of $F_{n}$ is similarly called irreducible if no proper free factor of $F_{n}$ is preserved (i.e., sent to a conjugate of itself ... remember we are talking about outer automorphisms). If $\phi$ is irreducible and all of its powers are irreducible, it is called an iwip (irreducible with irreducible powers), or a fully irreducible automorphism.

For fully reducible automorphisms Bestvina and Handel proved the existence of combinatorial structures on graphs, which they called train tracks by analogy with Thurston's train tracks for surfaces 13 . We will describe these structures and their relation to the given fully irreducible automorphism in the next section. The proof that train tracks exist was combinatorial and quite intricate. Bestvina was motivated to revisit the Lipschitz metric when he realized it could be used to give a conceptual geometric proof of the existence of these train tracks along the lines of Bers' proof in [6]. Here is a very rough sketch of the proof.

4.1. The basic trichotomy. We first divide elements $\phi$ of $\operatorname{Out}\left(F_{n}\right)$ into classes by looking at the smallest distance they can move points in $\mathrm{CV}_{n}$. Specifically, let $D=\inf _{\Gamma \in \mathrm{CV}_{n}} d(\Gamma, \Gamma \phi)$. Then $\phi$ is

- elliptic if $D=0$ and is realized,

- hyperbolic if $D>0$ and is realized,

- parabolic if $D$ is not realized.

Elliptic elements are the easiest to understand, since $\phi$ is elliptic if and only if it fixes a point $\Gamma \in \mathrm{CV}_{n}$, in which case an optimal difference of markings from $\Gamma$ to $\Gamma \phi=\Gamma$ is an isometry and so has finite order. 

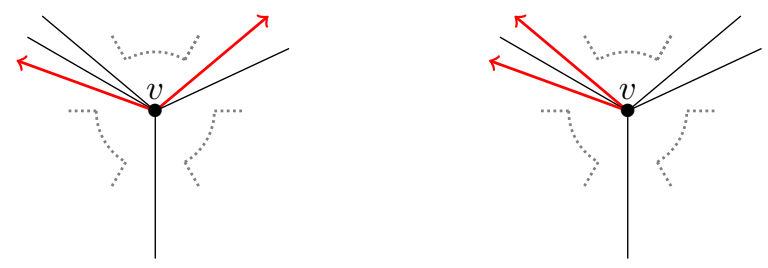

Figure 6. Gates at $v$ with a legal turn (left) and an illegal turn (right)

If $\phi$ is parabolic, consider any sequence of optimal maps $f_{k}: \Gamma_{k} \rightarrow \Gamma_{k}$ with $d\left(\Gamma_{k}, \Gamma_{k} \phi\right)$ converging to inf $d(\Gamma, \Gamma \phi)$. Using a compactness argument Bestvina shows that all but finitely many of these $\Gamma_{k}$ have a core subgraph whose edges are very small compared to the other edges. Since edges cannot get stretched by more than $\lambda=e^{D}$ by an optimal map, for $k$ large the small subgraph must be invariant under any difference of markings, so determines a proper free factor of $F_{n}$ which is invariant (up to conjugacy) under some power of $\phi$. We conclude that parabolic elements are reducible, so if $\phi$ is fully irreducible, it must be hyperbolic.

The key to understanding hyperbolic isometries is to find a point $\Gamma$ in $\mathrm{CV}_{n}$ which is moved a minimal distance and for which there is a particularly nice difference of markings map from $\Gamma$ to $\Gamma \phi$. Here "particularly nice" means $f: \Gamma \rightarrow \Gamma$ is an optimal map, the tension subgraph $\Delta=\Delta(f)$ is mapped into itself, and the restriction of $f$ to $\Delta$ is an extremely efficient type of map called a train track map. To explain what this means, we introduce a little terminology (see Figure 6).

- A train track structure on a graph $\Gamma$ is an equivalence relation on the directions at each vertex.

- Equivalence classes of directions at a vertex $v$ are called gates.

- A pair of directions at a vertex is called a turn.

- A turn is illegal if the directions are equivalent, i.e., belong to the same gate, and legal otherwise.

- A path in $\Gamma$ is legal if it does not take any illegal turns.

A map $f: \Delta \rightarrow \Delta$ is a train track map if there is a train track structure on $\Delta$ with the following properties:

- there are at least two gates at every vertex of $\Delta$,

- the image of each edge of $\Delta$ is a legal path, and

- the image of each legal turn in $\Delta$ is a legal turn.

If $\phi$ is a hyperbolic automorphism, there is a very elegant proof that such a "particularly nice" difference of markings exists, which we sketch in section 4.1.1 below. The tension graph $\Delta$ may well be a proper subgraph of $\Gamma$, in which case some power of $\phi$ is reducible. But if $\phi$ is fully irreducible, $\Delta$ must be all of $\Gamma$ and, after adjusting $f$ slightly to make vertices go to vertices, we have the following theorem, originally proved by Bestvina and Handel.

Theorem 4.1 ([13]). Let $\phi \in \operatorname{Out}\left(F_{n}\right)$ be fully irreducible. Then there is a graph $\Gamma$ and train track map $f: \Gamma \rightarrow \Gamma$ taking vertices to vertices and inducing $\phi$ on $\pi_{1}(\Gamma) \cong F_{n}$. 

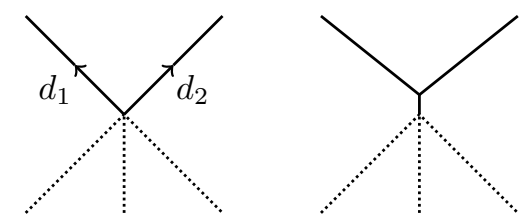

Figure 7. Folding an illegal turn

This theorem is the basis of a great deal of subsequent work on $\operatorname{Out}\left(F_{n}\right)$. It might be considered the "Golden Spike" uniting Thurston's theory of train tracks for surface automorphisms [39] with Stallings' theory of folding maps for automorphisms of free groups 37 .

4.1.1. Hyperbolic automorphisms and train tracks. Let $\phi$ be a hyperbolic automorphism, and let $\Gamma$ be a point in $\mathrm{CV}_{n}$ realizing $\inf _{\Gamma \in \mathrm{CV}_{n}} d(\Gamma, \Gamma \phi)=D>0$. Choose an optimal difference of markings $f: \Gamma \rightarrow \Gamma$, i.e., a difference of markings with stretch factor $\lambda=e^{D}>1$. By Proposition 3.2 we may assume the tension subgraph $\Delta=\Delta(f)$ is a core graph with at least two gates at every vertex. We define the complexity of such an $f$ in terms of $\Delta$, namely

$$
c(f)=(\text { rank of } \Delta,- \text { number of components of } \Delta) \text {. }
$$

Since $\Delta$ has no univalent vertices, removing an edge from $\Delta$ either reduces rank or increases the number of connected components, so decreases complexity.

Take an $f$ as above with minimal possible complexity. We want to prove that the restriction of $f$ to $\Delta$ is a train track map. We have to show that $f$ takes $\Delta$ to $\Delta$, maps each edge to a legal path, and takes legal turns to legal turns.

Step 1 . Suppose $f(\Delta) \not \subset \Delta$. Perturb $\Gamma$ by uniformly expanding $\Delta$ while shrinking the complement $\Gamma-\Delta$. Do this a very small amount, so that no edges are added to $\Delta$. Any edge of $\Delta$ which was mapped into $\Delta$ is still stretched by $\lambda$. But an edge of $\Delta$ whose image wanders outside of $\Delta$ will now be stretched by less than $\lambda$, so it will disappear from $\Delta$, contradicting minimality of $c(f)$. Note that $\Delta$ cannot disappear completely under our deformation: some edge must still be stretched by $\lambda$ since $\lambda$ is minimal for maps in the homotopy class of $f$. Thus $f(\Delta) \subset \Delta$.

Step 2. This step involves the notion of folding an illegal turn. If $\left\{d_{1}, d_{2}\right\}$ is a turn, folding by $\epsilon$ means identifying initial segments in the directions $d_{1}$ and $d_{2}$ of length $\epsilon$, so that the "V" formed by $d_{1}$ and $d_{2}$ becomes a "Y" with a very short stem (see Figure (7). If $\left\{d_{1}, d_{2}\right\}$ is illegal, then the directions $f\left(d_{1}\right)$ and $f\left(d_{2}\right)$ agree, so that $f$ induces a map on the folded graph if $\epsilon$ is small enough. We call the induced map an $\epsilon$-fold of $f$.

Suppose there is an edge $e$ such that $f(e)$ makes an illegal turn. Fold that turn slightly, but not enough to add edges to $\Delta$. In the folded map, the image of $e$ is homotopic to a shorter path so $e$ "drops out of $\Delta$ " (actually, $c(f)$ decreases), contradicting minimality.

Step 3. Suppose there is a legal turn $\left\{d_{1}, d_{2}\right\}$ that gets mapped to an illegal turn $\left\{f\left(d_{1}\right), f\left(d_{2}\right)\right\}$. Fold $\left\{f\left(d_{1}\right), f\left(d_{2}\right)\right\}$ slightly (without adding edges to $\left.\Delta\right)$. Then $\left\{d_{1}, d_{2}\right\}$ becomes illegal and we do not have to worry about where it gets mapped. But we do have to worry about the fact that formerly legal turns may have become 
illegal, and we may have decreased the number of gates at other vertices of $\Delta$, possibly down to a single gate at some vertex $v$. If we have done that, though, we could fix it with a homotopy of $f$ which moves the image of $v$ slightly into the image of its adjacent edges. This makes $\Delta$ smaller (decreases the complexity of $f$ ), again contradicting minimality.

And that's it.

The best ways to get from $A$ to $B$ are those that involve minimal tension.

\section{Geodesics in the Lipschitz Metric}

Although the Lipschitz metric is not symmetric, one could obtain a genuine metric by simply symmetrizing it. Francaviglia and Martino [17] studied basic properties of both the Lipschitz metric and its symmetized version, and found that the unsymmetrized version has a number of advantages over the symmetrized one. Perhaps the greatest of these is that $\mathrm{CV}_{n}$ is geodesically complete in the unsymmetrized version, but is not in the symmetrized one. In fact we can exhibit and analyze specific geodesics between any two points, and understanding the behavior of these geodesics is the key to many applications of the metric.

5.1. Recognizing geodesics. A path $\gamma(t)$ is a geodesic if and only if the triangle inequality is an equality for any three points along the path, i.e., for any $t_{0} \leq t_{1} \leq t_{2}$,

$$
d\left(\gamma\left(t_{0}\right), \gamma\left(t_{1}\right)\right)+d\left(\gamma\left(t_{1}\right), \gamma\left(t_{2}\right)\right)=d\left(\gamma\left(t_{0}\right), \gamma\left(t_{2}\right)\right)
$$

In terms of the Lipschitz distance this translates to: If $\Gamma_{t}$ is a path in $\mathrm{CV}_{n}$ such that the "same" loop is maximally stretched from each point on the path to any point further along, then $\Gamma_{t}$ is a geodesic. Here loops are the same if they represent the same conjugacy class of $F_{n}$.

5.2. Example: straight line in a simplex. Suppose $\Gamma$ and $\Gamma^{\prime}$ are points in $\mathrm{CV}_{n}$ which are in the same simplex, i.e., they differ only by the lengths of the graph's edges. Then we can define a path between them by simply scaling all edge lengths linearly. Lengths of edges are stretched (or shrunk) at a constant rate all along this path, so the same loop is maximally stretched all along the path. Therefore, by the criterion stated in the last subsection, the path is a geodesic.

5.3. Example: folding line. Let $f: \Gamma \rightarrow \Gamma^{\prime}$ be an optimal difference of markings with the additional property that the tension subgraph is all of $\Gamma$. Then there is a special class of geodesics from $\Gamma$ to $\Gamma^{\prime}$ called folding lines. We have already seen tiny folds in the proof of the classification of automorphisms when we identified initial segments in the directions of an illegal turn.

The map $f$ induces a train track structure on $\Gamma$ which puts directions at $v$ into the same gate if they map to the same direction at $f(v) \in \Gamma^{\prime}$. (Note that this does not mean that $f$ is a train track map!) If $f$ is not an isometry, there must be at least one illegal turn, and as before we can identify initial segments in the directions of this illegal turn to get an induced "folded" map. The folded map is still optimal, and the tension subgraph is still the entire domain, so unless the folded map is an isometry, we may continue the path by folding some more. If we do this long enough, we will eventually arrive at $\Gamma^{\prime}$. We think of this as a continuous process and call this a folding line from $\Gamma$ to $\Gamma^{\prime}$. Figure 8 illustrates the graphs along a 

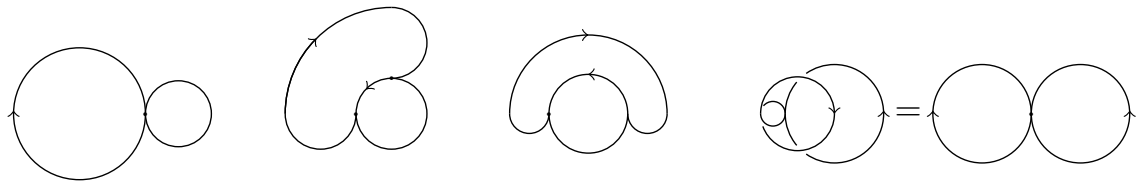

Figure 8. Folding a graph using an optimal map

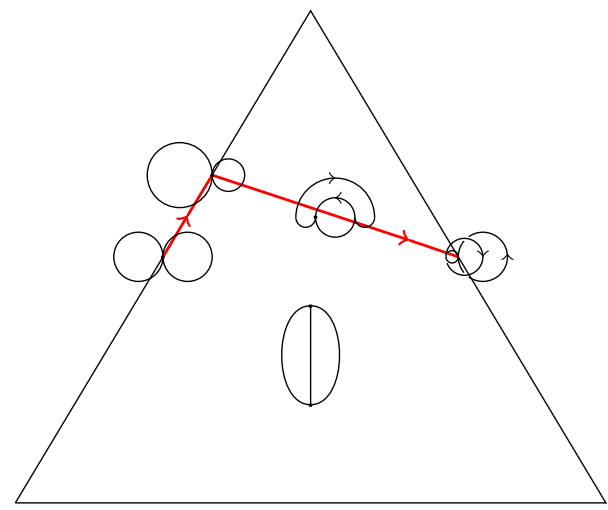

FiguRE 9. Folding line

folding line from a rank 2 rose to its image under the automorphism sending $a$ to $a b$ and fixing $b$, where $a$ and $b$ are the generators of $F_{2}$ represented by the simple loops in the starting graph.

If the tension subgraph is not all of $\Gamma$, we may need to change the edge lengths of $\Gamma$ (expanding the edges in $\Delta$ and contracting the others) to make $f$ into an optimal map with $\Delta=\Gamma$. Since this only moves us within a simplex, we may accomplish this by traveling along a straight line, as in Example 5.2. The composition of this straight line with a folding line as above is a geodesic in $\mathrm{CV}_{n}$, and we will abuse terminology by calling this a folding line as well. Figure 9 illustrates this with the folding line of Figure 8

5.4. So many roads. There are many geodesics between two given points. If the points are in the same simplex, we do not have to take the straight line between them, as long as we keep the same loop maximally stretched while traveling. If the two points are related by an optimal difference of markings whose tension subgraph is the entire graph, we can start by folding at any one of the illegal turns to get a folding line. To get a more canonical geodesic between two points, we could take the straight line until the tension subgraph is the whole graph, then take the "greedy" folding line which folds all illegal turns simultaneously at the same rate. But that still does not give unique paths, because there can be different optimal maps between two points.

5.5. Asymmetry of geodesics. Since there are so many geodesics between points, one might hope that there is some path which is a geodesic in both directions. Coulbois and Wiest demolished this hope by a simple example in rank 2 (see [17]). Figure 10 shows two marked theta graphs $\Gamma_{1}$ and $\Gamma_{2}$, contained in adjacent triangles 


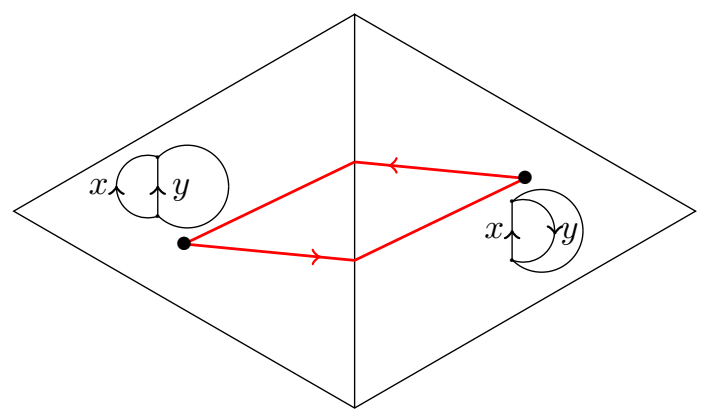

FiguRE 10. Asymmetry of geodesics

of $\mathrm{CV}_{2}$. The edges of $\Gamma_{1}$ have lengths $1 / 3,1 / 6$, and $1 / 2$ from left to right, and the edges of $\Gamma_{2}$ have lengths $1 / 6,1 / 3$, and $1 / 2$. Any geodesic from $\Gamma_{1}$ to $\Gamma_{2}$ must contain a rose $R$ in the common face of the triangles, with edge lengths $\ell$ and $1-\ell$ for some $\ell$. Since this is a geodesic, we must have $d\left(G_{1}, R\right)+d\left(R, G_{2}\right)=d\left(G_{1}, G_{2}\right)$. Calculating these distances (using candidate loops) subject to this constraint shows that $\ell$ must be equal to $5 / 8$. Calculating in the other direction gives $\ell=3 / 8$, i.e., no geodesic from $\Gamma_{1}$ to $\Gamma_{2}$ is equal to any geodesic from $\Gamma_{2}$ to $\Gamma_{1}$.

There are many ways to travel from here to there, and the way back might avoid them all.

5.6. Geodesics in the thick part. Traversing a geodesic from $x$ to $y$ backwards gives a path which needn't be a geodesic, and in fact may be arbitrarily far from any geodesic from $y$ to $x$. However, this cannot happen if the geodesic stays away from the "thin part" of $\mathrm{CV}_{n}$. A marked graph $\Gamma$ is said to be in the $\epsilon$-thin part if some embedded loop in $\Gamma$ has length at most $\epsilon$. Not surprisingly, the complement of the $\epsilon$-thin part is called the $\epsilon$-thick part. In a paper which analyzes the asymmetry of the Lipschitz metric quite precisely, Algom-Kfir and Bestvina prove the following statement.

Theorem 5.1 ([3]). If a geodesic $\gamma$ from $x$ to $y$ stays in the $\epsilon$-thick part of $\mathrm{CV}_{n}$, then the same path traversed backwards is a quasi-geodesic, i.e., has a parametrization which distorts arc length by a uniformly bounded amount, where the bound depends only on $\epsilon$.

\section{Hyperbolicity}

Gromov introduced a notion of negative curvature for metric spaces which is now known as Gromov hyperbolicity, or simply hyperbolicity. Hyperbolicity is a coarse invariant, meaning that a metric space which is "close" to a hyperbolic metric space must also be hyperbolic. The technically correct notion here is quasi-isometry. Metric spaces $X$ and $Y$ are quasi-isometric if there is a map $f: X \rightarrow Y$ which distorts distances by a bounded amount and is coarsely surjective, i.e., every point of $Y$ is within bounded distance of some $f(x)$. If a metric space is quasi-isometric to a hyperbolic space, then it is itself hyperbolic.

Gromov pointed out that the Cayley graph of a group with respect to any finite generating set can be regarded as a metric space and showed that Cayley graphs associated to different generating sets are quasi-isometric so that hyperbolicity of 
the Cayley graph is a group invariant of finitely generated groups. He went on to show that hyperbolicity implies many strong algebraic properties of a group, so it is a very useful thing to establish.

By the Swartz-Milnor lemma one can show that a group is hyperbolic by finding any proper and cocompact action on a hyperbolic metric space, so it is tempting to check the (proper) action of $\operatorname{Out}\left(F_{n}\right)$ on $\mathrm{CV}_{n}$ for these properties. We immediately come up short however, because Outer space with the Lipschitz metric is not hyperbolic and the action of $\operatorname{Out}\left(F_{n}\right)$ is not cocompact. The fact that the action is not cocompact is easily fixed: replace $\mathrm{CV}_{n}$ by its simplicial closure $\overline{\mathrm{CV}}_{n}$. In addition to cocompactness we have (amazingly) gained hyperbolicity, by a deep theorem of Handel and Mosher:

Theorem 6.1 ([22]). The simplicial closure $\overline{\mathrm{CV}}_{n}$ of Outer space is hyperbolic.

Here the metric on $\overline{\mathrm{CV}}_{n}$ which makes it into a hyperbolic metric space is not an extension of the Lipschitz metric, but rather the metric obtained by regarding each simplex as a regular Euclidean simplex with edges of length 1.

Remark 6.2. $\overline{\mathrm{CV}}_{n}$ is also known as the free splitting complex, since vertices can be re-interpreted as actions on trees with one edge-orbit and trivial edge stabilizer and by Bass-Serre theory such an action corresponds to a splitting of $F_{n}$ as a free product or HNN extension. As we remarked in Section $2.3, \overline{\mathrm{CV}}_{n}$ is also the same as the sphere complex $\mathcal{S}_{n}$.

We now have a cocompact action of $\operatorname{Out}\left(F_{n}\right)$ on a hyperbolic metric space, but $\operatorname{Out}\left(F_{n}\right)$ is not a hyperbolic group. One algebraic consequence of hyperbolicity is that a hyperbolic group cannot contain free abelian subgroups of rank 2 , and it is easy to find large abelian subgroups in $\operatorname{Out}\left(F_{n}\right)$. The problem here is that the action of $\operatorname{Out}\left(F_{n}\right)$ on $\overline{\mathrm{CV}}_{n}$ is not proper, in fact it is easy to see that the stabilizer of every simplex at infinity is infinite.

This mirrors the situation for the Teichmüller space of a surface $S$ with its action by the mapping class $\operatorname{group} \operatorname{Mod}(S)$ of isotopy classes of diffeomorphisms of $S$. There is no hyperbolic metric on Teichmüller space which is $\operatorname{Mod}(S)$-invariant, but there is a related hyperbolic complex whose simplices are "on the boundary at infinity" of Teichmüller space called the curve complex $\mathcal{C}(S)$. $\operatorname{Mod}(S)$ acts by isometries on $\mathcal{C}(S)$; this action is not proper, but one can nevertheless use hyperbolicity of the curve complex to establish properties of the mapping class group. The key to these applications lies in the fact, due to Masur and Minsky, that distances in (the non-hyperbolic group) $\operatorname{Mod}(S)$ can be approximately measured by adding up distances in all (hyperbolic!) curve complexes associated to all subsurfaces of $S$ 32,33 .

Masur and Minsky used the Teichmüller metric on Teichmüller space in an essential way in all of their work, and most of the current work on the geometry of Outer space is inspired by attempts to adapt their methods to the context of Outer space and the Lipschitz metric. In the next sections we will describe some of this work.

\section{Contracting AXes FOr IWIPS}

Although Teichmüller space is not hyperbolic, geodesics in certain directions behave like geodesics in a hyperbolic space. Think of a geodesic in the hyperbolic 
plane and a ball of any radius disjoint from the geodesic: the closest-point projection of the ball onto the geodesic has uniformly bounded diameter. (This is in marked contrast with the behavior of balls and geodesics in the Euclidean plane!) A geodesic with this property in any metric space is called a contracting geodesic, and all geodesics in a hyperbolic space are contracting. Minsky showed that in Teichmüller space with the Teichmüller metric, the axis of any pseudo-Anosov mapping class is also a contracting geodesic 34.

Recall that for $\operatorname{Out}\left(F_{n}\right)$ an iwip (or fully irreducible automorphism) is in some ways analogous to a pseudo-Anosov mapping class, and that iwips have train track representatives (Theorem 4.1). A train-track representative for an iwip $\phi$ can be thought of as a difference of markings from a point $\Gamma$ of $\mathrm{CV}_{n}$ to its image $\Gamma \phi$. Since a train track representative is an optimal map, there is a folding line from $\Gamma$ to $\Gamma \phi$, whose image under $\phi$ is a folding line from $\Gamma \phi$ to $\Gamma \phi^{2}$. For such a $\Gamma$ and $\phi$ the concatenation of these two folding lines is itself a folding line, so by iterating this procedure, we obtain a bi-infinite (forward) geodesic connecting the images of $\Gamma$ under all powers of $\phi$. We call this geodesic an axis for $\phi$.

Algom-Kfir extended the Teichmüller analogy by proving that the axis of an iwip in $\mathrm{CV}_{n}$ is coarsely contracting in the Lipschitz metric. More precisely, she defined a coarse projection from all of Outer space onto the axis and proved that the image of a ball sufficiently far away from the axis has uniformly bounded diameter [1. Making sense of this takes a great deal of care and requires developing coarse versions of many techniques. Problems include the fact that the axis of an iwip is not unique (train tracks themselves are not unique), distances in one direction do not agree with distances in the other, the projection of a point in $\mathrm{CV}_{n}$ is most naturally a bounded set instead of a single point, etc. But hyperbolicity is only a coarse notion anyway, and Algom-Kfir's theorem implies that many of the geometric benefits hyperbolicity confers are still available in the direction of an iwip. For example quasi-geodesics with endpoints on the axis of an iwip stay within bounded distance of that axis, geodesics in $\mathrm{CV}_{n}$ diverge at least quadratically fast, and the asymptotic cone of $\mathrm{CV}_{n}$ contains many cut-points.

\section{USING THE LIPSCHITZ METRIC TO PROVE HYPERBOLICITY OF THE FREE FACTOR COMPlex (BestVina AND FEIGHN)}

A foundational result in all of Masur and Minsky's work is the fact that the curve complex is hyperbolic. For $\operatorname{Out}\left(F_{n}\right)$ there are actually several reasonable candidates for an analog of the curve complex. We have already mentioned the simplicial closure $\overline{\mathrm{CV}}_{n}$ but another natural choice is the complex of conjugacy classes of free factors $\mathcal{F} \mathcal{F}_{n}$. A vertex of $\mathcal{F F}_{n}$ is a free factor of $F_{n}$, i.e., a subgroup $A$ generated by part of a basis for $F_{n}$. The conjugacy classes of free factors $A$ and $B$ are connected by an edge if some conjugate of $A$ is a subgroup of $B$. A set of conjugacy classes form the vertices of a simplex if and only if every pair in the set is connected by an edge.

Bestvina and Feighn proved that $\mathcal{F F}_{n}$ is hyperbolic by adapting Masur and Minsky's methods to Outer space with the Lipschitz metric [10]. This was followed quite soon by Handel and Mosher's proof that $\overline{\mathrm{CV}}_{n}$ is hyperbolic, by much more combinatorial methods 22]. (Later Kapovich and Rafi showed that in fact hyperbolicity of $\mathcal{F F}_{n}$ can be derived from hyperbolicity of $\overline{\mathrm{CV}}_{n}$ [30.) In their work on 
subfactor projections Bestvina and Feighn needed stronger results than were available from Handel and Mosher's proof, so they showed that the Lipschitz metric and folding lines could be used to streamline the Handel-Mosher proof and, in the process, find more quasi-geodesics [11. In the next few paragraphs I will attempt to give some of the ideas involved in their proof for $\mathcal{F F}_{n}$.

The proof depends on a criterion developed by Brian Bowditch for proving that a space $X$ is hyperbolic [14]. Bowditch shows that it is sufficient to find a constant $C$ and a family of paths in $X$ satisfying the following conditions:

(1) Any two points $x, y \in X$ are coarsely joined by one of the paths, i.e., there is a path in the family with one endpoint within $C$ of $x$ and the other within $C$ of $y$.

(2) Paths in the family which begin at nearby points and end at nearby points stay within Hausdorff distance $C$ of one another.

(3) Paths in the family from $x$ to $y$ are within Hausdorff distance $C$ of paths in the family from $y$ to $x$.

(4) The paths satisfy the "thin triangles" condition, i.e., for any three points $x, y$, and $z$, a path in the family from $x$ to $z$ is in the $C$-neighborhood of the union of any paths in the family from $x$ to $y$ and $y$ to $z$.

So to use Bowditch's criterion, we need to find a family of paths in $\mathcal{F F}_{n}$. We already have a family of preferred paths in $\mathrm{CV}_{n}$, namely folding lines. And it is easy to define a projection from $\mathrm{CV}_{n}$ to $\mathcal{F F}_{n}$ : for any marked graph $\Gamma=(g, \Gamma)$, consider the proper free factors of $F_{n}$ determined by subgraphs $\Gamma^{\prime}$ of $\Gamma$ (i.e., proper free factors of the form $\left.g_{*}^{-1}\left(\pi_{1}\left(\Gamma^{\prime}\right)\right)<\pi_{1}\left(R_{n}\right) \cong F_{n}\right)$; we can define a projection $\pi: \mathrm{CV}_{n} \rightarrow \mathcal{F F}_{n}$ by taking any one (or all) of them. The projection $\pi$ is coarsely well defined since the free factors determined by any two subgraphs are within distance 4 in the free factor complex; furthermore $\pi$ is coarsely Lipschitz. Given any folding line $\gamma=\left\{\Gamma_{t}\right\}$ the vertices $\pi\left(\Gamma_{t}\right)$ of $\mathcal{F F}_{n}$ change at discrete times, and we can connect the dots to form a path $\pi(\gamma)$ in $\mathcal{F F}_{n}$. Paths constructed in this way will be our preferred paths in $\mathcal{F} \mathcal{F}_{n}$.

To see that any two free factors are coarsely connected by a preferred path, find points in $\mathrm{CV}_{n}$ such that the first free factor is realized as a subgraph of the first marked graph and the second as a subgraph of the second. Connect these points by a folding line and project this line to the free factor complex.

To check the next two conditions of Bowditch's criterion, Bestvina and Feighn define a projection in the other direction, from $\mathcal{F F}_{n}$ onto a fixed folding line $\gamma$. Morally, the idea is to project a free factor $A$ to the interval of $\gamma$ in which $A$ is "smallest" and show that interval has uniformly bounded size.

One way of measuring the size of a free factor $A$ in a marked graph $\Gamma$ is to lift $\Gamma$ to the unprojectivized Outer space of $A$ and measure the volume of the lift. To define this lift, think of a point in $\mathrm{CV}_{n}$ as an action of $F_{n}$ on a metric tree. The subgroup $A<F_{n}$ also acts on this tree, so there is a minimal subtree (consisting of the axes of all elements of $A$ ) which is a point in the unprojectivized Outer space for $A$. The volume of the lift is then the volume of the quotient of this minimal subtree by the action of $A$. An equivalent way to describe this quotient is as the core of the cover of $\Gamma$ corresponding to the subgroup $A<F_{n}=\pi_{1}(\Gamma)$.

Unfortunately minimizing volume in this naive way does not result in projections of uniformly bounded size, and it is not so easy to define a projection from $\mathcal{F} \mathcal{F}_{n}$ to the folding line $\gamma$ with the desired properties. The definition that Bestvina and 
Feighn come up with does begin by lifting each marked graph $\Gamma_{t}$ in $\gamma$ to a point in the Outer space of $A$, as we have described. But instead of measuring volume they measure the lengths of legal and illegal paths in the lifts.

Recall that a folding line $\gamma$ is constructed using an optimal map from its starting point $\Gamma_{0}$ to its endpoint $\Gamma_{L}$ (after adjusting the edge-lengths of $\Gamma_{0}$ ), and that the induced map from any point $\Gamma_{t}$ along the path to $\Gamma_{L}$ is still optimal. Each of these optimal maps determines a train track structure on its domain $\Gamma_{t}$, where directions at a vertex are in the same gate if they are mapped to the same direction in $\Gamma_{L}$. Since the turns of the $A$-lift of $\Gamma_{t}$ cover turns in $\Gamma_{t}$, we can pull back the train-track structure on $\Gamma_{t}$ to define a train track structure on its $A$-lift, and hence define the notions of legal and illegal paths in this lift. Bestvina and Feighn show that in the forward direction along $\gamma$ these $A$-lifts have longer and longer immersed legal paths, whereas in the backward direction legal paths tend to develop illegal segments. Left and right projections of $A$ to $\gamma$ can now be defined by specifying the times at which the lifts develop long immersed legal paths or at which they have long immersed paths containing only very short legal segments. These left and right projections are then shown have bounded distance in the Lipschitz metric.

Let $\ell_{\gamma}: \mathcal{F F}_{n} \rightarrow \gamma$ be the left projection just defined, so we now have the following diagram of maps.

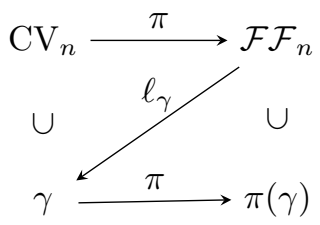

The composition $\pi \circ \ell_{\gamma}$ is a coarse Lipschitz retraction from all of $\mathcal{F F}_{n}$ to $\pi(\gamma)$. In particular, the restriction of this retraction to $\pi(\gamma)$ is Lipschitz, so $\pi(\gamma)$ is an unparametrized quasi-geodesic and our collection of paths $\{\pi(\gamma)\}$ satisfies conditions (2) and (3) of Bowditch's hyperbolicity criterion.

To complete the proof that $\mathcal{F F}_{n}$ is hyperbolic, it remains to establish the thin triangles condition. This is again done by analyzing legal and illegal paths in the graphs $\Gamma_{t}$ which occur along folding lines.

The proof that all of this actually works is a technical tour-de-force relying on an intimate understanding of the evolution of legal systems in folding lines.

\section{Subfactor projections (Bestvina and Feighn)}

A second critical element of Masur-Minsky's theory relating the geometry of curve complexes to that of Teichmüller space is the notion of subsurface projections. For a subsurface $A$, the subsurface projection $\pi_{A}$ is a map from the curve complex of $S$ to the curve complex of $A$. It is defined by intersecting curves in $S$ with $A$, then closing up these intersections if necessary using arcs in $\partial A$ to form simple closed curves in $A$. If $B$ is another subsurface, then $\pi_{A}(B)$ denotes $\pi_{A}$ applied to the boundary curves of $B$.

Recall that there is a natural projection from the Teichmüller space of $S$ to the curve complex $\mathcal{C}(S)$ which picks out a shortest curve. As one travels along a Teichmüller geodesic, one may not be progressing at all in $\mathcal{C}(S)$ (e.g., if a single curve $\alpha$ remains shortest along the entire geodesic), but there has to be some subsurface 
$A$ such that progress is being made in $\mathcal{C}(A)$. Masur and Minsky prove that in fact distance in Teichmüller space can be approximately measured by adding up distances in all possible subsurface projections. This has myriad applications, one of which is an application to the dimension of the mapping class group.

9.1. Is the asymptotic dimension of $\operatorname{Out}\left(F_{n}\right)$ finite? The usual (covering) dimension of a metric space is definitely not a quasi-isometry invariant (for example, all compact metric spaces are quasi-isometric!) Gromov defined a new type of dimension called asymptotic dimension which is invariant under quasi-isometry, so in particular defines an invariant for finitely generated groups. The asymptotic dimension of any compact set is zero, the asymptotic dimension of $\mathbb{R}^{n}$ is equal to $n$ and the asymptotic dimension of a tree is equal to one. Even these calculations are non-trivial, however, and in general it is very difficult to compute asymptotic dimension or even show that it is finite. Bestvina, Bromberg, and Fujiwara did manage to show that the mapping class group $\operatorname{Mod}(S)$ has finite asymptotic dimension [8]. Their strategy was to use curve complexes and subsurface projections to construct a product of hyperbolic spaces on which each infinite order element of $\operatorname{Mod}(S)$ acts with positive translation length. Bell and Fujiwara had previously proved that curve complexes have finite asymptotic dimension [5], and this can be used to show that this product space does as well. This is enough to conclude that $\operatorname{Mod}(S)$ has finite asymptotic dimension.

Bestvina and Feighn have begun to fill in the pieces of this scheme in the case of $\operatorname{Out}\left(F_{n}\right)$ by defining an analog of subsurface projections called subfactor projections. These project one free factor onto the free factor complex of another one [1]. (Technically, Bestvina and Feighn's projections land in the free splitting complex of the second free factor, but this can be followed by projection to the free factor complex, which is a uniformly Lipschitz map. Very recently, Sam Taylor has studied a more natural version of subfactor projection which projects one free factor directly onto the free factor complex of another [38].) These projections satisfy the conditions necessary for constructing a product of hyperbolic spaces with an $\operatorname{Out}\left(F_{n}\right)$-action, as in the case of the mapping class group. However, one can only conclude that exponentially growing automorphisms act with positive translation length, not that all infinite order ones do. This translates to the fact that these subfactor projections do not do a complete job of estimating distance in $\operatorname{Out}\left(F_{n}\right)$; adding up the distances in the free factor complexes of all subfactors gives a lower bound on the distance but not an upper bound. One might say that "not enough" projections have yet been defined to measure progress along a geodesic in $\mathrm{CV}_{n}$. This is a situation which will undoubtedly be remedied in the near future.

\section{REMARKS}

Some things can be simplified by regarding $\overline{\mathrm{CV}}_{n}$ as the sphere complex $\mathcal{S}_{n}$, including the proof that $\overline{\mathrm{CV}}_{n}$ is hyperbolic 28 .

Masur and Minsky's original theorem that the curve complex of a surface is hyperbolic now has a vastly simpler proof and the constant of hyperbolicity has been shown to be independent of the surface by several of groups of people, using new criteria for hyperbolicity and new combinatorial techniques; these simplifications may well be adaptable to $\operatorname{Out}\left(F_{n}\right)$. In general things are developing very rapidly in this subject. 


\section{Guide to the References}

There is quite a large literature by now concerning the geometry of $\mathrm{CV}_{n}$. The references in this article contain a selection of these papers, which fall roughly into the following categories.

11.1. Classics and background. Material relevant to this article from mapping class groups and Teichmüller theory can be found in Thurston's iconic article on the geometry of 3-manifolds [39, his preprint on the Lipschitz metric on Teichmüller space [40, Masur and Minsky's original articles on the curve complex [32, 33, Bowditch's proof that the curve complex is hyperbolic [14], and Bers' article classifying surface automorphisms [4. For automorphisms of free groups and Outer space Stallings' article on folding [37] and Thurston's proof of the Bounded Cancellation Lemma [15] are seminal. In addition there are the Culler and Vogtmann article introducing Outer space [16], Bestvina and Handel's proof of the Scott conjecture which introduces train tracks, and Hatcher's interpretation of Outer space in terms of sphere systems [27].

11.2. Basics of the Lipschitz metric on outer space. A good introduction to the Lipschitz metric can be found in Bestvina's PCMI notes [7]. For further details see Algom-Kfir and Bestvina's article on asymmetry of the Lipschitz metric [3], Algom-Kfir's paper on the metric completion of $\mathrm{CV}_{n}$ [2], and Francoviglia and Martino's original paper on the Lipschitz metric [17.

11.3. Lines in $\mathrm{CV}_{n}$. There are quite a few notions of "good paths" in Outer space. These include the folding paths described in this article, for which Bestvina's PCMI notes [7] are a good introduction. Other paths are the lines of minima studied by Hammenstadt [18, the axis bundles defined by Handel and Mosher in [20, the related fold lines defined in their proof that $\overline{\mathrm{CV}}_{n}$ is hyperbolic [20], and surgery paths in the sphere system model of $\mathrm{CV}_{n}$ as first defined by Hatcher in 27] and further developed for example in Horbez's paper [29].

11.4. Hyperbolicity of $\operatorname{Out}\left(F_{n}\right)$-complexes. The first $\operatorname{Out}\left(F_{n}\right)$ complex shown to be hyperbolic was the one defined by Bestvina and Feighn in [9]. However, that complex was not canonically defined (it depended on the choice of an iwip), and not all iwips had unbounded orbits. Bestvina and Feighn's proof that the free factor complex is hyperbolic appeared next [10, followed quickly by Handel and Mosher's proof that $\overline{\mathrm{CV}}_{n}$ is hyperbolic [22. Hilion and Horbez considerably simplified the Handel-Mosher proof by using the sphere complex description of $\overline{\mathrm{CV}}_{n}$ [28], and Kapovich and Rafi show that hyperbolicity of $\overline{\mathrm{CV}}_{n}$ implies hyperbolicity of $\mathcal{F F}_{n}$ [30]. In [36] Sabalka and Savchuk show that one must include non-separating spheres in the sphere complex, otherwise the complex is not hyperbolic.

11.5. Projections and asymptotic dimension. Bestvina, Bromberg, and Fujiwara showed that the mapping class group of a surface has finite asymptotic dimension [8]. This built on previous work of Bell and Fujiwara proving that the curve complex has finite asymptotic dimension [5] and of Bestvina and Fujiwara constructing quasi-morphisms on mapping class groups using subsurface projections [12. Efforts to extend these ideas to Outer space include Bestvina and Feighn's work on subsurface projections [10, 11, Sam Taylor's simpler notion of subfactor projections [38], Sabalka and Savchuk's submanifold projections 35], and the work 
of Hamenstadt and Hensel on subsurface projections [19] from the point of view of sphere complexes.

11.6. Related work using more combinatorial methods. Handel and Mosher's extensive work on automorphisms of free groups avoids using the Lipschitz metric, developing instead more combinatorial methods based on the theory of train tracks [21. Their most recent contribution is a series of four substantial papers which study the subgroup structure of $\operatorname{Out}\left(F_{n}\right)[23$ 26].

\section{ACKNOWLEDGMENTS}

The author would like to thank Mladen Bestvina, Mark Feighn, Saul Schleimer, and Sam Taylor for very helpful conversations during the preparation of this article.

\section{ABOUt THE AUTHOR}

Karen Vogtmann is the Goldwin Smith Professor of Mathematics at Cornell University and a professor of mathematics at the University of Warwick. She works in geometric group theory and cohomology of groups and pays special attention to automorphism groups.

\section{RefERENCES}

[1] Yael Algom-Kfir, Strongly contracting geodesics in outer space, Geom. Topol. 15 (2011), no. 4, 2181-2233, DOI 10.2140/gt.2011.15.2181. MR.2862155

[2] Yael Algom-Kfir, The Metric Completion of Outer Space, arXiv:1202.6392.

[3] Yael Algom-Kfir and Mladen Bestvina, Asymmetry of outer space, Geom. Dedicata 156 (2012), 81-92, DOI 10.1007/s10711-011-9591-2. MR2863547

[4] Lipman Bers, An extremal problem for quasiconformal mappings and a theorem by Thurston, Acta Math. 141 (1978), no. 1-2, 73-98. MR0477161 (57 \#16704)

[5] Gregory C. Bell and Koji Fujiwara, The asymptotic dimension of a curve graph is finite, J. Lond. Math. Soc. (2) 77 (2008), no. 1, 33-50, DOI 10.1112/jlms/jdm090. MR2389915 (2009d:57037)

[6] Mladen Bestvina, A Bers-like proof of the existence of train tracks for free group automorphisms, Fund. Math. 214 (2011), no. 1, 1-12, DOI 10.4064/fm214-1-1. MR2845630 (2012m:20046)

[7] Mladen Bestvina, PCMI Lectures on the geometry of Outer space, (2014), 1-34.

[8] Mladen Bestvina, Kenneth Bromberg, and Koji Fujiwara. Constructing group actions on quasi-trees and applications to mapping class groups, arXiv:1006.1939.

[9] Mladen Bestvina and Mark Feighn, A hyperbolic Out $\left(F_{n}\right)$-complex, Groups Geom. Dyn. 4 (2010), no. 1, 31-58, DOI 10.4171/GGD/74. MR2566300 (2011a:20052)

[10] Mladen Bestvina and Mark Feighn, Hyperbolicity of the complex of free factors, arXiv:1107.3308. (2011).

[11] Mladen Bestvina and Mark Feighn, Subfactor projections, arXiv:1211.1730.

[12] Mladen Bestvina and Koji Fujiwara, Quasi-homomorphisms on mapping class groups, Glas. Mat. Ser. III 42(62) (2007), no. 1, 213-236, DOI 10.3336/gm.42.1.15. MR.2332668 (2008k:57002)

[13] Mladen Bestvina and Michael Handel, Train tracks and automorphisms of free groups, Ann. of Math. (2) 135 (1992), no. 1, 1-51, DOI 10.2307/2946562. MR1147956 (92m:20017)

[14] Brian H. Bowditch, Intersection numbers and the hyperbolicity of the curve complex, J. Reine Angew. Math. 598 (2006), 105-129, DOI 10.1515/CRELLE.2006.070. MR2270568 (2009b:57034)

[15] Daryl Cooper, Automorphisms of free groups have finitely generated fixed point sets, J. Algebra 111 (1987), no. 2, 453-456, DOI 10.1016/0021-8693(87)90229-8. MR916179 (89a:20024)

[16] Marc Culler and Karen Vogtmann, Moduli of graphs and automorphisms of free groups, Invent. Math. 84 (1986), no. 1, 91-119, DOI 10.1007/BF01388734. MR830040 (87f:20048) 
[17] Stefano Francaviglia and Armando Martino, Metric properties of Outer space, Publ. Mat. 55 (2011), no. 2, 433-473, DOI 10.5565/PUBLMAT_55211_09. MR2839451 (2012j:20128)

[18] Ursula Hamenstädt, Lines of minima in Outer space, Duke Math. J. 163 (2014), no. 4, 733-776, DOI 10.1215/00127094-2429807. MR.3178431

[19] Ursula Hamenstädt and Sebastian Hensel, Spheres and Projections for Out $\left(F_{n}\right)$, arXiv:1109.2687.

[20] Michael Handel and Lee Mosher, Axes in Outer space, Mem. Amer. Math. Soc. 213 (2011), no. 1004, vi+104, DOI 10.1090/S0065-9266-2011-00620-9. MR.2858636

[21] Michael Handel and Lee Mosher, Lipschitz retraction and distortion for subgroups of Out $\left(F_{n}\right)$, Geom. Topol. 17 (2013), no. 3, 1535-1579, DOI 10.2140/gt.2013.17.1535. MR3073930

[22] Michael Handel and Lee Mosher, The free splitting complex of a free group, I: hyperbolicity, Geom. Topol. 17 (2013), no. 3, 1581-1672, DOI 10.2140/gt.2013.17.1581. MR3073931

[23] Michael Handel and Lee Mosher, Subgroup decomposition in $\operatorname{Out}\left(F_{n}\right)$, Part I: Geometric Models, arXiv:1302.2378.

[24] Michael Handel and Lee Mosher, Subgroup decomposition in $\operatorname{Out}\left(F_{n}\right)$, Part II: A relative Kolchin theorem, arXiv:1302.2379.

[25] Michael Handel and Lee Mosher, Subgroup decomposition in $\operatorname{Out}\left(F_{n}\right)$, Part III: Weak attraction theory, arXiv:1306.4712.

[26] Michael Handel and Lee Mosher, Subgroup decomposition in $\operatorname{Out}\left(F_{n}\right)$, Part IV: Relatively irreducible subgroups, arXiv:1306.4711.

[27] Allen Hatcher, Homological stability for automorphism groups of free groups, Comment. Math. Helv. 70 (1995), no. 1, 39-62, DOI 10.1007/BF02565999. MR1314940 (95k:20030)

[28] Arnaud Hilion and Camille Horbez, The hyperbolicity of the sphere complex via surgery paths, arXiv:1210.6183.

[29] Camille Horbez, Sphere paths in outer space, Algebr. Geom. Topol. 12 (2012), no. 4, 24932517, DOI 10.2140/agt.2012.12.2493. MR3020214

[30] Ilya Kapovich and Kasra Rafi, On hyperbolicity of free splitting and free factor complexes, arXiv:1206.3626.

[31] Francois Laudenbach, Sur les 2-spheres d'une varièté de dimension 3, Ann. of Math. 97 (1973) 57-81. MR0314054

[32] Howard A. Masur and Yair N. Minsky, Geometry of the complex of curves. I. Hyperbolicity, Invent. Math. 138 (1999), no. 1, 103-149, DOI 10.1007/s002220050343. MR 1714338 (2000i:57027)

[33] H. A. Masur and Y. N. Minsky, Geometry of the complex of curves. II. Hierarchical structure, Geom. Funct. Anal. 10 (2000), no. 4, 902-974, DOI 10.1007/PL00001643. MR1791145 (2001k:57020)

[34] Yair N. Minsky, Quasi-projections in Teichmüller space, J. Reine Angew. Math. 473 (1996), 121-136, DOI 10.1515/crll.1995.473.121. MR1390685(97b:32020)

[35] Lucas Sabalka and Dmitri Savchuk, Submanifold projection, arXiv:1211.3111.

[36] Lucas Sabalka and Dmitri Savchuk, On the geometry of a proposed curve complex analogue for Out $\left(F_{n}\right)$, arXiv:1007.1998.

[37] John R. Stallings, Finite graphs and free groups, Combinatorial methods in topology and algebraic geometry (Rochester, N.Y., 1982), Contemp. Math., vol. 44, Amer. Math. Soc., Providence, RI, 1985, pp. 79-84, DOI 10.1090/conm/044/813103. MR813103

[38] Samuel J. Taylor, A note on subfactor projections, Algebr. Geom. Topol. 14 (2014), no. 2, 805-821, DOI 10.2140/agt.2014.14.805. MR.3159971

[39] William P. Thurston, The geometry and topology of three-manifolds, Princeton Univ. Press (1978).

[40] William P. Thurston, Minimal stretch maps between hyperbolic surfaces, arXiv:9801.039.

UNIVERSiTy OF WARWICK AND CORNELl UNIVERSITY

E-mail address: k.vogtmann@warwick.ac.uk 